\title{
A heuristic approach for component scheduling on a high-speed PCB assembly machine
}

\author{
William $\mathrm{Ho}^{1, *}$, Ping $\mathrm{Ji}^{2}$ and Yongzhong $\mathrm{Wu}^{2}$ \\ ${ }^{1}$ Operations and Information Management Group \\ Aston Business School, Aston University \\ Birmingham B4 7ET, United Kingdom \\ Email: w.ho@aston.ac.uk; Fax: +44 (0)121 2045271; Tel: +44 (0)121 2043342 \\ ${ }^{2}$ Department of Industrial and Systems Engineering, \\ The Hong Kong Polytechnic University, \\ Hung Hom, Kowloon, Hong Kong
}

\begin{abstract}
The collect-and-place machine is one of the most widely used placement machines for assembling electronic components on the printed circuit boards (PCBs). Nevertheless, the number of researches concerning the optimisation of the machine performance is very few. This motivates us to study the component scheduling problem for this type of machines with an objective of minimising the total assembly time. The component scheduling problem is an integration of the component sequencing problem, that is the sequencing of component placements, and the feeder arrangement problem, that is the assignment of component types to feeders. To solve the component scheduling problem efficiently, a hybrid genetic algorithm is developed in this paper. A numerical example is used to compare the performance of algorithm with different component grouping approaches and different population sizes.
\end{abstract}

Keywords: Printed circuit board assembly; Collect-and-place machines; Component scheduling; Hybrid genetic algorithm

* Corresponding author 


\section{Introduction}

The extensive applicability in numerous contemporary electronic products, including mobile phones, televisions, and computers, has placed an unprecedented demand for PCBs nowadays. PCB assembly, which is the process of assembling electronic components on a PCB, can be classified into two categories: plated-through-hole (PTH) technology and surface mount technology (SMT). Requirements from consumers, such as smaller product size and greater function and reliability, have forced the SMT to replace the PTH technology. Among several assembly operations in an SMT assembly line, the component placement process is generally the most time-consuming (Ong and Khoo 1999, Ong and Tan 2002), frequently a bottleneck (Ong and Tan 2002, Csaszar et al. 2000), and determines the line cycle time (Wilhelm and Tarmy 2003). To increase the throughput rate of a PCB manufacturing company, the time spent on assembling all electronic components to a board using an SMT placement machine must always be kept to minimum. Ball and Magazine (1988) suggested that the assembly time is dependent on the component sequencing problem (i.e. which component is placed first, second, and so on) and the feeder arrangement problem (i.e. which component type is stored in which feeder). Therefore, in this paper, both problems are taken into consideration in order to minimise the total assembly time.

In PCB assembly, there are various types of SMT placement machines. Each type of machines possesses its own characteristics and operation. Among them, the three most widely used types are the pick-and-place, the chip shooter, and the collect-and-place (CAP) machines. For the former two types of machines, the component scheduling problem, which is an integration of both component sequencing and feeder arrangement problems, has been studied thoroughly (Ho and Ji 2003-2006). However, to our best knowledge, there are only three publications in the international literature studying the component scheduling problem for the CAP machine. Altinkemer et al. (2000) formulated a mathematical model for the problem in 
which two rigid assumptions were made. First, they assumed that the CAP machine is equipped with one revolver head only. This assumption is not realistic because it consists of two or even more revolver heads. Second, they assumed that the revolver head can pick up components of the same type in the same tour only. However, the capacity of the revolver head may exceed the number of components of the same type. On this occasion, extra tours are incurred because of that assumption. As a consequence, the total distance traveled by the revolver head or the time spent on assembling the components is longer. Kazaz and Altinkemer (2003) extended the mathematical model formulated in Altinkemer et al. (2000) by allowing components of the same type to be stored in multiple feeders. Similarly, the authors focused on the CAP machine with one revolver head only. Instead of applying the mathematical modeling approach, Grunow et al. (2004) proposed a heuristic approach to solve the component scheduling problem for the CAP machine with one revolver head. However, the component sequencing and feeder arrangement problems were solved separately. Altinkemer et al. (2000) proved that the efficiency of component placement process in the SMT environment is also dependent on a feeder to hold which types of components besides the pick and placement sequence. If the component sequencing and feeder arrangement problems are not solved simultaneously, it can cause significant deterioration in the machines performance.

All the above publications focused on the one-head CAP machines. Lack of research contributions in optimizing the multi-head CAP machine performance is the primal motivation for undergoing this project. To eliminate their drawbacks, in this paper, both component sequencing and feeder arrangement problems are solved simultaneously instead of separately as Grunow et al. (2004) did. In addition, it is valid that the revolver head can pick up components of the different types instead of the same type only as Altinkemer et al. (2000) and Kazaz and Altinkemer (2003) proposed. 
The individual component sequencing and feeder arrangement problems are already very sophisticated, and hard to solve (Ho and Ji 2003-2006). Hence, the problem being studied in this paper is extremely complicated and very challenging. Moreover, the problem sizes in the real cases prohibit the effective use of mathematical modeling approaches. So, a genetic algorithm is developed to solve the component scheduling problem for the CAP machine.

This paper is structured as follows. Section 2 discusses both characteristics and operation sequence of the CAP machine. Section 3 describes a genetic algorithm hybridised with several improved heuristics for the component scheduling problem. Section 4 compares the performance of the algorithm using two different component grouping methods, and studies the effect of population sizes on the solutions' quality. Finally, Section 5 draws conclusions and discusses possible future work.

\section{The collect-and-place machine}

The type of placement machines being studied is called the CAP machine, which is one of the three main kinds of machines used for component placements in the SMT environment besides the pick-and-place and chip shooter machines as mentioned in Section 1. Because of its unique configuration, the CAP machine combines the advantages of both pick-and-place and chip shooter machines. It not only can assure high placement accuracy but also can achieve high placement speed. This type of machines, as illustrated in figure 1, has multiple stationary feeders (each feeder can hold components of the same type only), a stationary X-Y table securing a PCB, and two revolver heads with several vacuum nozzles (normally, 12). While one head is collecting components from its adjacent various stationary feeders, the other head is placing components on the stationary board, and vice versa.

[Insert figure 1 about here] 
Consider a PCB with $n$ components to be assembled by a CAP machine with two revolver heads. Each head has $N_{n}$ nozzles to collect and place components. Note that the head is always fully loaded with $N_{n}$ components except for the final assembly tour. So, the number of assembly tours required for assembling all components or $N_{t}=\operatorname{ceil}\left(n / N_{n}\right)$. For an assembly tour, say $t$, the placement sequence is the same as the pick-up sequence. It is because the revolver head performs stepwise rotational movements only in one or forward direction while reverse rotation is not allowed. For instance, there are $n$ components on a PCB to be assembled by a CAP machine, in which each revolver head possesses $n / 2$ nozzles. Therefore, two assembly tours are incurred. Each revolver head is responsible for an assembly tour. In this case, the operation sequence of both revolver heads, say $H_{A}$ and $H_{B}$, in the CAP machine is described as follows. $H_{A}$ starts from its original location or starting point, moves to a fixed feeder that carries components, picks up a component from the feeder, indexes one step. After that, $H_{A}$ remains stationary to the previous feeder if the next component is the same type with the previous one or moves to another feeder if it is different with the previous one to pick up another component. This operation procedure is repeated until $H_{A}$ finishes collecting all the first half components. After collecting, $H_{A}$ moves to the desired placement location on the fixed PCB, and places the components one by one at their predetermined locations. When $H_{A}$ is placing the first half components on the $\mathrm{PCB}, H_{B}$ is collecting the remaining half components from the fixed feeders adjacent to $H_{B}$. At last, $H_{B}$ traverses to the PCB, and places the components sequentially on the PCB.

\section{A hybrid genetic algorithm}

Genetic algorithms (GAs) have been applied to solve a wide variety of hard optimisation problems successfully, including the traveling salesman problem (TSP), the quadratic assignment problem (QAP), and the minimum spanning tree problem (Gen and Cheng 1997). 
In addition, the merits of GAs, such as simplicity, ease of operation, and flexibility, encourage us to apply it to tackle the component scheduling problem for the CAP machine. Ideas of the general GAs are described thoroughly by Goldberg (1989).

The component scheduling problem can be regarded as a combination of TSP and QAP because it was proved that the component sequencing and feeder arrangement problems are very similar to the TSP and QAP, respectively (Ho and Ji 2003-2006). Due to the problem complexity, a simple GA may not perform well in this situation. The GA developed in this paper is therefore hybridised with several heuristics in order to improve the solution further, which is a prevalent approach nowadays (Luu et al. 2002).

The HGA for the component scheduling problem, as shown in figure 2, is described as follows. After the parameters, including iteration number, population size, crossover rate, and mutation rate, have been set, the HGA generates the initial chromosomes or solutions to the problem. Each chromosome contains three links. The first link representing the sequence of component placements is generated using the nearest neighbor heuristic (NNH). The second and the third links indicating the feeder arrangements for two stand-alone feeders (one is near $H_{A}$ while the other one is adjacent to $H_{B}$ ) are generated randomly. After that, the iterated swap procedure (ISP) is used to improve the three links. Each chromosome is then measured by an evaluation function. The roulette wheel method is adopted to select some chromosomes for the genetic operations, including the modified order crossover, heuristic mutation, and inversion mutation. After an offspring is produced, all its three links are improved by the ISP again. The fitness of the offspring will be measured and may become a member of the population if it possesses a relatively good quality. These steps form an iteration, and then the roulette wheel selection is performed again to start the next iteration. The HGA will not stop unless the predetermined number of iterations is conducted.

[Insert figure 2 about here] 


\subsection{Encoding}

The detailed algorithm is discussed in the current and subsequent subsections. The first decision needs to be made when implementing GAs is which representation of the chromosomes (i.e. encoding) is designed. In this paper, the path representation is selected to encode the chromosomes of the component scheduling problem for the CAP machine. The idea of the path representation is that the components are listed in the order in which they are placed. A chromosome with the three-link path representation is illustrated in figure 3 . Link 1 represents the sequence of component placements, Link 2 denotes the assignment of component types to feeders next to $H_{A}$, whereas Link 3 indicates the assignment of component types to another feeders close to $H_{B}$.

[Insert figure 3 about here]

Consider a PCB with 10 components (numbered as $1,2, \ldots, 10$ ) to be assembled by a CAP machine with two revolver heads $\left(H_{A}\right.$ and $\left.H_{B}\right)$. Each revolver head has five nozzles. Therefore, each head is responsible for collecting and placing five components. If the sequence of placements starts with component 1 , then components $2,5,10,4,9,7,3,6$, and finally component 8, its sequence can be represented as (1 251049736 8). It is the first link or Link 1 in a chromosome as shown in figure 3. The number inside the bracket in Link 1 represents the component type. For example, component 1 of type 4 is placed first by $H_{A}$, whereas component 9 of type 1 is placed in advance by $H_{B}$. Besides, Links 2 and 3 show the assignments of components to feeders. For instance, component type 4 is located at feeder $A 2$ or $f_{A 2}$ in Link 2 (i.e. feeder 2 close to $H_{A}$ ), and feeder $B 1$ or $f_{B 1}$ in Link 3 (i.e. feeder 1 beside $H_{B}$ ). In this case, $H_{A}$ has to visit $f_{A 2}$ initially to pick up a component of type 4 for the first component. The entire assembly sequence of each revolver head is shown in the following:

1st Operation $\quad H_{A}$ collects: $\quad$ starting point of $H_{A} \rightarrow f_{A 2} \rightarrow f_{A 1} \rightarrow f_{A 2} \rightarrow f_{A 3} \rightarrow f_{A 4}$;

$H_{B}$ waits. 
2nd Operation $\quad H_{A}$ places: $\quad f_{A 4} \rightarrow c_{1} \rightarrow c_{2} \rightarrow c_{5} \rightarrow c_{10} \rightarrow c_{4}$;

$$
H_{B} \text { collects: } \quad \text { starting point of } H_{B} \rightarrow f_{B 2} \rightarrow f_{B 1} \rightarrow f_{B 2} \rightarrow f_{B 3} \rightarrow f_{B 4} \text {. }
$$

3rd Operation $H_{A}$ finishes;

$$
H_{B} \text { places: } \quad f_{B 4} \rightarrow C_{9} \rightarrow c_{7} \rightarrow c_{3} \rightarrow c_{6} \rightarrow c_{8} .
$$

\subsection{Improved heuristics}

In the HGA, two heuristics are adopted to improve the solution, including the NNH and ISP. First, the NNH is used to generate an initial solution for the first link only, which is the sequence of component placements. The principle of the $\mathrm{NNH}$ is to start with the first component randomly, then to select the next component as close as possible to the previous one from those unselected components to form the placement sequence until all components are selected.

Second, the ISP is used to improve all the three links of each initial solution as well as each offspring generated by the three genetic operators further. The principle of the ISP, as illustrated in figure 4, is very similar to that of the 2-opt local search heuristic, except that some instead of all two swaps are examined. Thus, the ISP is more efficient than the 2-opt local search heuristic. The procedure of the ISP is as follows (Ho and Ji 2003-2006):

Step 1: $\quad$ Select two genes randomly from a link of a parent.

Step 2: $\quad$ Exchange the positions of the two genes to form an offspring.

Step 3: $\quad$ Swap the neighbors of the two genes to form four more offspring.

Step 4: Evaluate all offspring and find the best one.

Step 5: If the best offspring is better than the parent, replace the parent with the best offspring and go back to Step 1. Otherwise, stop.

[Insert figure 4 about here] 


\subsection{Evaluation}

Without evaluation, it is impossible to compare and then find the best solution. Therefore, evaluation is often regarded as the most important element of GAs. For the CAP machine, the evaluation or fitness function used is the summation of all dominating times of the operations. Actually, the number of operations is equal to $N_{t}+1$. Referring to the 10 -component problem described in Section 3.1, the number of operations required to assemble all components is three because $N_{t}=\operatorname{ceil}\left(n / N_{n}\right)=\operatorname{ceil}(10 / 5)=2$. The movements of $H_{A}$ and $H_{B}$ in each of the three operations have been shown. In the first operation, the dominating time is exactly the time used by $H_{A}$ to collect the first five components from feeder $A$ because $H_{B}$ remains stationary [equation (1)]. In the second operation, the dominating time is the longest one among the time used by $H_{A}$ to place the first five components and the time spent by $H_{B}$ to collect the last five components from feeder $B$ [equation (2)]. Finally, the dominating time in the third operation is simply the time spent by $H_{B}$ to assemble the last five components on the PCB because $H_{A}$ finishes its assembly already [equation (3)].

Let $\operatorname{eval}\left(X_{h}\right)$ be the fitness function for chromosome $X_{h}$ in the component scheduling problem. In case $N_{t}=2$, then

$$
\begin{aligned}
\operatorname{eval}\left(X_{h}\right)= & T\left[c_{A}(0), f_{A}\left(a_{1}\right)\right]+\sum_{i=a_{1}}^{N_{c c(1)}-1} T\left[f_{A}(i), f_{A}(i+1)\right] \\
& +\max \mid\left\langle\left\{\left\{f_{A}\left[N_{c c(1)}\right], c_{A}\left(a_{1}\right)\right\}+\sum_{i=a_{1}}^{N_{c c(1)}-1} T\left[c_{A}(i), c_{A}(i+1)\right]\right\rangle,\right| \\
& +T\left\{f_{B}\left[N_{c c(2)}\right], c_{B}\left(a_{2}\right)\right\}+\sum_{i=a_{2}}^{N_{c c(2)}-1} T\left[c_{B}(i), c_{B}(i+1)\right]
\end{aligned}
$$

where

$T(a, b)$ is the longest traveling time for moving from point $a$ to point $b$; 
$T(a, b)=\left\{\begin{array}{l}\max \left(\frac{\left|x_{b}-x_{a}\right|}{V_{x}}, \frac{\left|y_{b}-y_{a}\right|}{V_{y}}, t_{r}\right) \text { if the head moves from a feeder to the other one } \\ \max \left(\frac{\left|x_{b}-x_{a}\right|}{V_{x}}, \frac{\left|y_{b}-y_{a}\right|}{V_{y}}\right) \text { otherwise. }\end{array}\right.$

$V_{x}$ and $V_{y}$ are the speed of the revolver head in $x$ and $y$ directions, respectively;

$t_{r}$ is the time for the revolver head to index one step;

$c_{A}(i)$ and $c_{B}(i)$ are the locations on the PCB for the $i$ th components placed by $H_{A}$ and $H_{B}$, respectively;

$f_{A}(i)$ and $f_{B}(i)$ are the locations of feeder $A$ and feeder $B$ for the $i$ th components, respectively;

$n$ is the number of components on a PCB;

$N_{n}$ is the number of nozzles in a revolver head;

$N_{t}$ is the number of assembly tours required for assembling all components;

$$
N_{t}=\operatorname{ceil}\left(n / N_{n}\right)
$$

$t$ is the tour number;

$$
t=1,2, \ldots, N_{t}
$$

$N_{c(t)}$ is the number of components collected and placed in tour $t$;

$$
N_{c(t)}=\min \left[N_{n}, N_{c r(t-1)}\right]
$$

$N_{c r(t)}$ is the number of components remained after tour $t$;

$$
N_{c r(t)}=N_{c r(t-1)}-N_{c(t)}
$$

$N_{c c(t)}$ is the cumulative number of components after tour $t$;

$$
N_{c c(t)}=N_{c c(t-1)}+N_{c(t)} ;
$$

$a_{t}$ is the first component in tour $t$;

$$
a_{t}=N_{n}(t-1)+1
$$

$c_{A}(0)$ and $c_{B}(0)$ refers to the starting points of $H_{A}$ and $H_{B}$, respectively. 
In case $N_{t}=3$, then

$$
\begin{aligned}
\operatorname{eval}\left(X_{h}\right)= & T\left[c_{A}(0), f_{A}\left(a_{1}\right)\right]+\sum_{i=a_{1}}^{N_{c c(1)}-1} T\left[f_{A}(i), f_{A}(i+1)\right] \\
& +\max \mid\left\langle\left\{\begin{array}{l}
\left.\left.\left.\mid f_{A}\left[N_{c c(1)}\right], c_{A}\left(a_{1}\right)\right\}+\sum_{i=a_{1}}^{N_{c c(1)}-1} T\left[c_{A}(i), c_{A}(i+1)\right]\right\rangle,\right\rangle \\
\left.T\left[c_{B}(0), f_{B}\left(a_{2}\right)\right]+\sum_{i=a_{2}}^{N_{c c(2)}-1} T\left[f_{B}(i), f_{B}(i+1)\right]\right\}
\end{array}\right.\right. \\
& +\max \mid\left\langle\begin{array}{l}
\left.T\left\{c_{A}\left[N_{c c(1)}\right], f_{A}\left(a_{3}\right)\right\}+\sum_{i=a_{3}}^{N_{c c(3)}-1} T\left[f_{A}(i), f_{A}(i+1)\right]\right\rangle \\
\left.T\left\{f_{B}\left[N_{c c(2)}\right], c_{B}\left(a_{2}\right)\right\}+\sum_{i=a_{2}}^{N_{c c(2)}-1} T\left[c_{B}(i), c_{B}(i+1)\right]\right\rangle
\end{array}\right\rangle \\
& +T\left\{f_{A}\left[N_{c c(3)}\right], c_{A}\left(a_{3}\right)\right\}+\sum_{i=a_{3}}^{N_{c c(3)}-1} T\left[c_{A}(i), c_{A}(i+1)\right]
\end{aligned}
$$

The number of operations required to assemble all components is four if $N_{t}=3$. The dominating times of the four operations are listed in the equations (4) to (7) accordingly.

\subsection{Selection}

The roulette wheel approach (Gen and Cheng 1997) is adopted in order to select some chromosomes to undergo genetic operations. The approach is based on an observation that a roulette wheel has a section allocated for each chromosome in the population, and the size of each section is proportional to the chromosome's fitness. The fitter the chromosome, the higher the probability of being selected. It is true that the roulette wheel selection mechanism chooses chromosomes probabilistically, instead of deterministically. For example, although one chromosome has the highest fitness, there is no guarantee it will be selected. The only thing that is certain is that on the average a chromosome will be chosen with the probability proportional to its fitness. 


\subsection{Genetic operations}

The genetic search progress is obtained by two essential genetic operations: exploitation (or intensification) and exploration (or diversification). Generally, the crossover operator exploits a better solution, whereas the mutation operator explores a wider search space. The genetic operators used in the algorithm for the component scheduling problem are one crossover and two mutations. Nowadays, GAs developed especially for the PCB assembly problems are not limited to using one crossover and one mutation (Ong and Khoo 1999, Ong and Tan 2002, Ho and Ji 2003-2006). Three links in a chromosome are required to perform these genetic operations. The number of chromosomes selected to perform the crossover and mutation operations depends on the crossover and mutation rates, respectively.

3.5.1 The modified order crossover. As shown in figure 5, a modified version of the classical order crossover operator is used. Two offspring will be generated at each time. The procedure of the modified order crossover operation is as follows (Ho and Ji 2003-2006):

Step 1: $\quad$ Select a substring from the first parent randomly.

Step 2: $\quad$ Produce a protochild by copying the substring into the corresponding positions in the protochild.

Step 3: Find the gene right prior to the first gene of the substring from the second parent. If the gene is one of the genes in the substring, go to Step 4. Otherwise, place it in front of the substring in the protochild.

Step 4: $\quad$ Find the gene right behind the last gene of the substring from the second parent. If the gene is one of the genes in the substring, go to Step 5. Otherwise, place it just after the substring in the protochild.

Step 5: Delete those genes that are already in the protochild from the second parent. The resulted genes, that is, the genes not in the protochild yet, form a sequence. 
Step 6: Place the genes into the unfilled positions of the protochild from the left to the right according to the resulted sequence of genes in Step 5 to produce an offspring.

Step 7: $\quad$ Repeat Step 1 to Step 6 to produce the second offspring by exchanging the two parents.

[Insert figure 5 about here]

3.5.2 The heuristic mutation. As shown in figure 6, a heuristic mutation operator is used to promote diversity of the population. All neighbors generated are used as the offspring. The procedure of the heuristic mutation operation is as follows (Ho and Ji 2003-2006):

Step 1: $\quad$ Pick up three genes in a parent at random.

Step 2: Generate neighbors for all possible permutations of the selected genes, and all neighbors generated are regarded as the offspring.

Three genes are selected in step 1 because two genes has only one variation (one offspring) while more than three genes will generate too many offspring and it will take a very long time for computation.

[Insert figure 6 about here]

3.5.3 The inversion mutation. The inversion operator is a mutation operation because it is very similar to the heuristic mutation that operates with one chromosome only. Thus, it lacks interchange of the characteristics between chromosomes. The principle of the inversion mutation operation is straightforward. As shown in figure 7, it simply selects a substring from a parent and flips it to form an offspring.

[Insert figure 7 about here] 


\section{Result analysis}

The performance of the HGA was evaluated using a PCB example. The example, as shown in tables 1 and 2, has 50 components with 10 different component types. The parameters of the HGA for the component scheduling problem are: population size $=25$, iteration number $=1000$, crossover rate $=0.4$, and mutation rate $=0.2$. Therefore, five pairs of chromosome are selected to perform the modified order crossover operation, whereas five chromosomes perform the heuristic mutation and inversion mutation operations. The total number of offspring produced per iteration will be 40 (10 from the modified order crossover operation, 25 from the heuristic mutation operation, and 5 from the inversion mutation operation).

[Insert table 1 about here]

[Insert table 2 about here]

\subsection{Component grouping: random vs. location}

The first computational study is to compare the performance of algorithm with different component grouping approaches, including random grouping and location grouping. Component grouping can be regarded as the head assignment problem. Actually, this is a problem of determining which revolver head places which components. The idea of the first approach, called random grouping, is that any sequence of component placements is permitted. Components no matter located at anywhere on a PCB can be assembled by $H_{A}$ or $H_{B}$. Conversely, in location grouping, $H_{A}$ must assemble those components located adjacent to it. The remaining components are assigned to $H_{B}$ for assembling.

The comparison of results is shown in Table 3. For population size $=25$, it is found that the performance of the HGA with location grouping (i.e. HGA2) is superior to that with random grouping (i.e. HGA1) in terms of the total assembly time. First, HGA2 generated a better initial solution than HGA1, 41.0833 vs. 43.9167 seconds. Second, the final best solution 
yielded by HGA2 is much better than that obtained by HGA1, 33.5833 vs. 37.5 seconds. HGA2 can generate better solutions because each revolver head is only permitted to assemble those components located adjacent to it. This can significantly reduce the distance traveled by the heads, and hence the total assembly time is shorter.

[Insert table 3 about here]

\subsection{Population size: 5 vs. 25}

Besides investigating the effect of the component grouping approaches, the effect of the population size on the performance of HGA is studied. Population size plays a vital role because it determines both efficiency and effectiveness of the HGA. The larger the population size, the higher the chance of yielding the optimal solution or a better solution. Nevertheless, larger population size spends longer times for computation and takes more computer storage space. To identify the effect, the HGAs (i.e. HGA1 and HGA2) are run with the 50-component problem again using a smaller population size: 5 . Figures 8 and 9 show the effect of population size on the performance of HGA1 and HGA2, respectively. The experimental results have been summarised in table 3.

According to figures 8 and 9, the curves representing the population size of 25 are lower than that representing the population size of 5. This phenomenon shows that the HGAs with larger population size can yield better initial and final solutions. This may be due to the fact that more offspring are produced at each iteration, 40 vs. 8 . It can therefore be proved that the quality of the HGAs' solution is highly related to the population size because it determines the number of chromosomes generated.

[Insert figure 8 about here]

[Insert figure 9 about here] 


\section{Conclusions}

In this paper, a heuristic approach was developed to solve the component scheduling problem for the collect-and-place machine with two revolver heads. The problem was proved to be very complex because it is a combination of the traveling salesman problem and the quadratic assignment problem, which are hard optimisation problems. To tackle the problem effectively and efficiently, a genetic algorithm hybridised with two improved heuristics were applied, including the nearest neighbor heuristic and the iterated swap procedure. The experimental findings proved that the performance of the algorithm with location grouping approach is superior to that of the algorithm with random grouping approach in terms of the total assembly time. In addition, it was proved that the algorithm with larger population size can yield better solutions.

Future studies should focus on the mathematical modeling approach so that the global optimal solutions can be yielded. Once it is obtained, the effectiveness of the proposed algorithms is known. Moreover, in the component scheduling problem, components of the same type are assigned to a single feeder only. In cases where the number of feeders available is greater than the number of component types, some components of the same type can be stored in more than one feeder subjecting to the feeder capacity. Therefore, the feeder arrangement problem no longer belongs to the category of 1-to-1 assignment problem. Besides the component sequencing and feeder arrangement problems, it is also necessary to determine which feeder a component should be retrieved from because more than one feeder hold the same type of components, that is, the component retrieval problem.

\section{Acknowledgements}

The authors wish to acknowledge The Hong Kong Polytechnic University for the financial support of the project (No. A-PG05). 


\section{References}

Altinkemer, K., Kazaz, B., Köksalan, M. and Moskowitz, H., Optimization of printed circuit board manufacturing: integrated modeling and algorithms. European Journal of Operational Research, 2000, 124, 409-421.

Ball, M.O. and Magazine, M.J., Sequencing of insertions in printed circuit board assembly. Operations Research, 1988, 36, 192-201.

Csaszar, P., Tirpak, T.M. and Nelson, P.C., Optimization of a high-speed placement machine using tabu search algorithms. Annals of Operations Research, 2000, 96, 125-147.

Gen, M. and Cheng, R., Genetic Algorithms and Engineering Design, 1997 (New York: Wiley).

Goldberg, D.E., Genetic Algorithms in Search, Optimization and Machine Learning, 1989 (New York: Addison-Wesley).

Grunow, M., Günther, H.O., Schleusener, M. and Yilmaz, I.O., Operations planning for collect-and-place machines in PCB assembly. Computers \& Industrial Engineering, 2004, 47, 409-429.

Ho, W. and Ji, P., Component scheduling for chip shooter machines: a hybrid genetic algorithm approach. Computers \& Operations Research, 2003, 30, 2175-2189.

Ho, W. and Ji, P., A hybrid genetic algorithm for component sequencing and feeder arrangement. Journal of Intelligent Manufacturing, 2004, 15, 307-315.

Ho, W. and Ji, P., A genetic algorithm to optimise the component placement process in PCB assembly. International Journal of Advanced Manufacturing Technology, 2005, 26, $1397-1401$.

Ho, W. and Ji, P., A genetic algorithm approach to optimising component placement and retrieval sequence for chip shooter machines. International Journal of Advanced Manufacturing Technology, 2006, 28, 556-560. 
Kazaz, B. and Altinkemer, K., Optimization of multi-feeder (depot) printed circuit board manufacturing with error guarantees. European Journal of Operational Research, 2003, 150, 370-394.

Luu, D.T., Bohez, E.L.J. and Techanitisawad, A., A hybrid genetic algorithm for the batch sequencing problem on identical parallel machines. Production Planning \& Control, 2002, 13, 243-252.

Ong, N.S. and Khoo, L.P., Genetic algorithm approach in PCB assembly. Integrated Manufacturing Systems, 1999, 10, 256-265.

Ong, N.S. and Tan, W.C., Sequence placement planning for high-speed PCB assembly machine. Integrated Manufacturing Systems, 2002, 13, 35-46.

SIEMENS, http://www.siplace.com, 2005.

Wilhelm, W.E. and Tarmy, P.K., Circuit card assembly on tandem turret-type placement machines. IIE Transactions, 2003, 35, 627-645. 


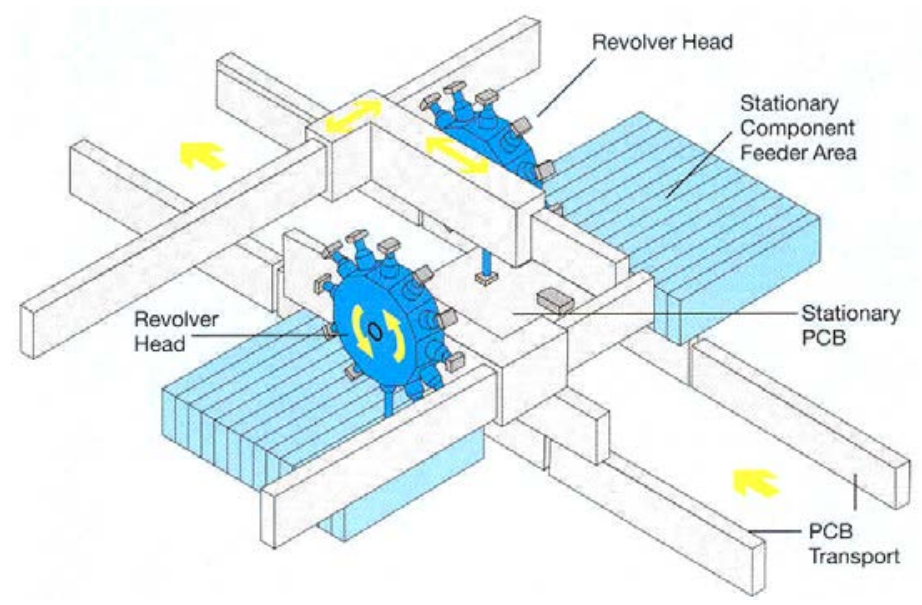

Figure 1. The schematic diagram of the collect-and-place machine (SIEMENS 2005). 


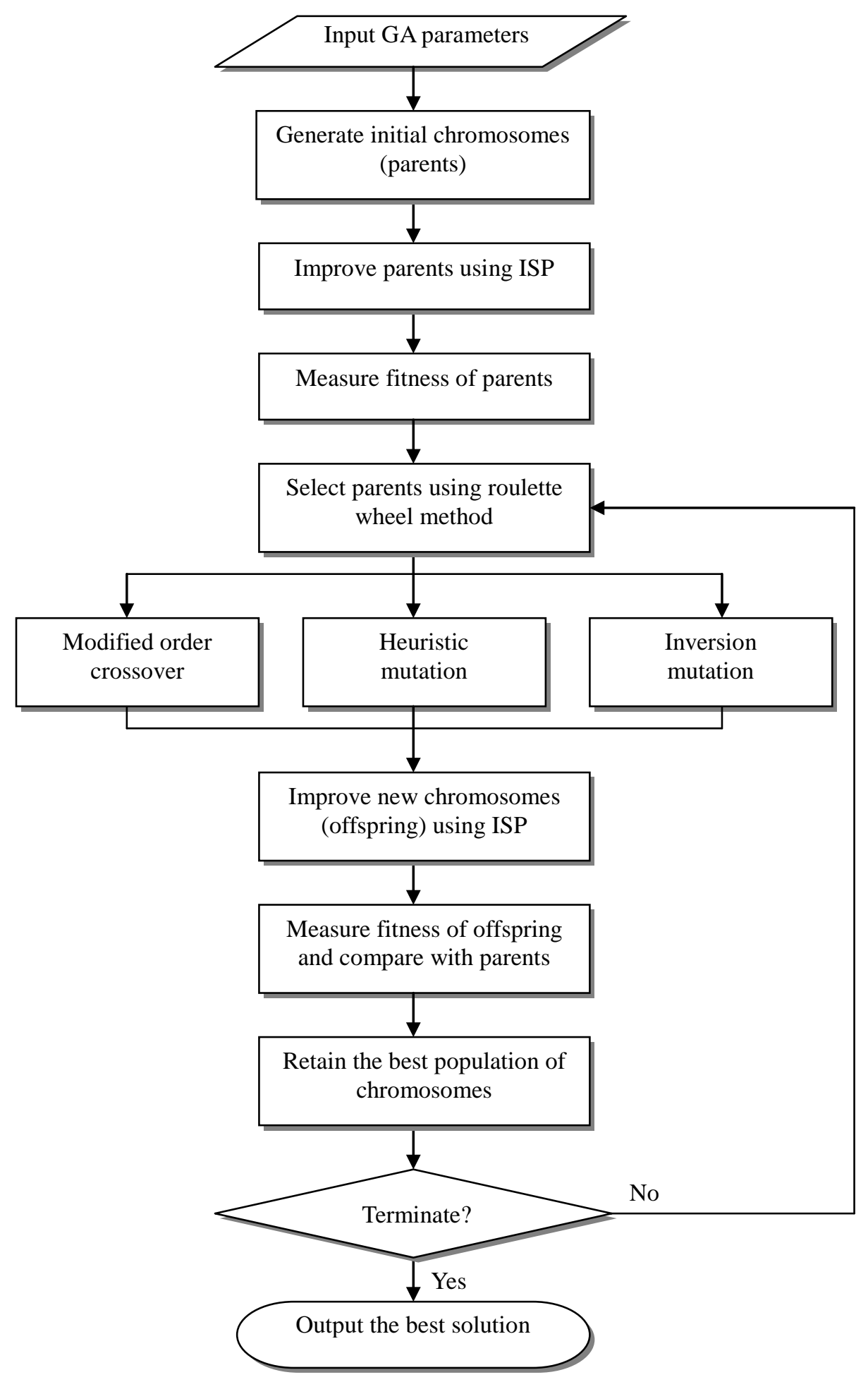

Figure 2. The flowchart of the HGA (Ho and Ji 2004-2006). 


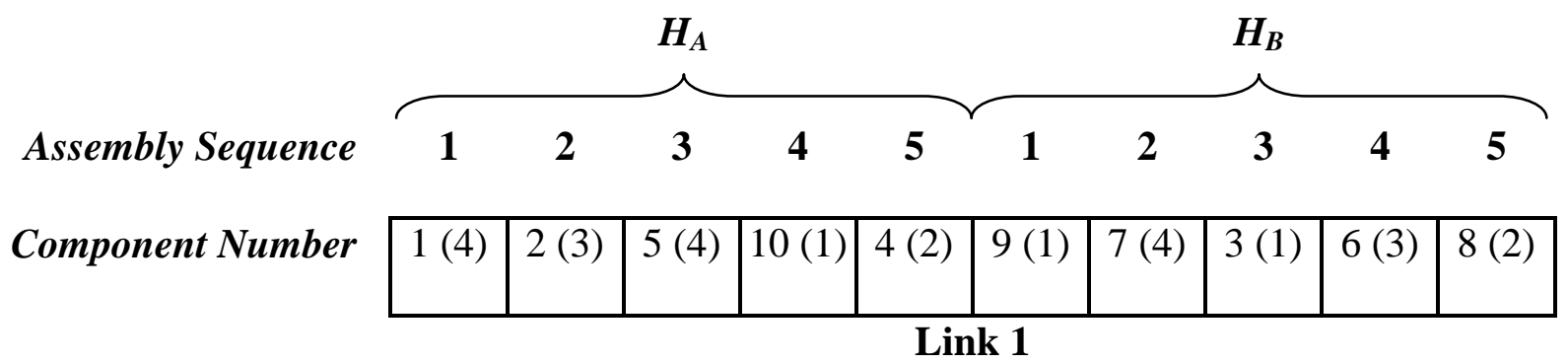

\begin{tabular}{c|c|c|c|c|}
\cline { 2 - 5 } Component Type & 3 & 4 & 1 & 2 \\
\cline { 2 - 6 } Feeder & A1 & A2 & A3 & A4
\end{tabular}

Link 2

\begin{tabular}{|c|c|c|c|}
\hline 4 & 1 & 3 & 2 \\
\hline B1 & B2 & B3 & B4
\end{tabular}

Link 3

Figure 3. The three-link representation for a chromosome. 
Select two genes randomly

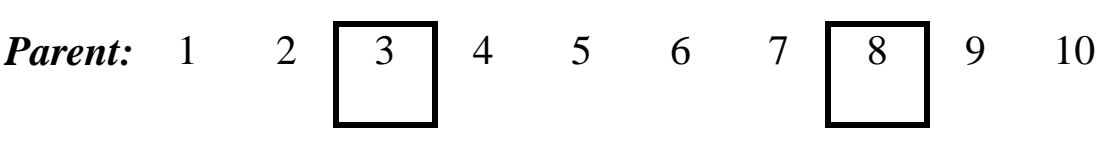

$\begin{array}{lllllllllll}\text { Offspring 1: } & 1 & 2 & \mathbf{8} & 4 & 5 & 6 & 7 & \mathbf{3} & 9 & 10 \\ \text { Offspring 2: } & 1 & \mathbf{8} & \mathbf{2} & 4 & 5 & 6 & 7 & 3 & 9 & 10 \\ \text { Offspring 3: } & 1 & 2 & \mathbf{4} & \mathbf{8} & 5 & 6 & 7 & 3 & 9 & 10 \\ \text { Offspring 4: } & 1 & 2 & 8 & 4 & 5 & 6 & \mathbf{3} & \mathbf{7} & 9 & 10 \\ \text { Offspring 5: } & 1 & 2 & 8 & 4 & 5 & 6 & 7 & \mathbf{9} & \mathbf{3} & 10\end{array}$

Figure 4. The iterated swap procedure. 


\section{Selected substring}

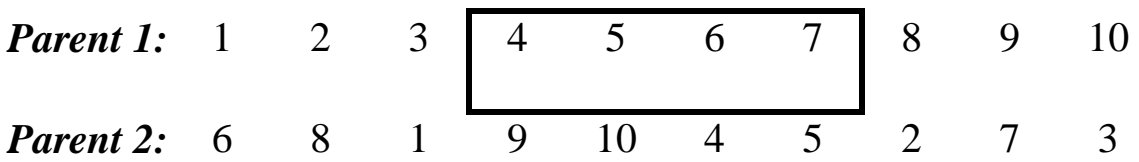

$\begin{array}{lllllllllll}\text { Offspring 1: } & 8 & 1 & 10 & \mathbf{4} & \mathbf{5} & \mathbf{6} & \mathbf{7} & 3 & 9 & 2\end{array}$

\section{Selected substring}

\begin{tabular}{|c|c|c|c|c|c|c|c|c|c|}
\hline Parent 2: & 6 & 8 & 1 & 9 & 10 & 4 & 5 & 2 & 7 \\
\hline Parent 1: & 1 & 2 & & 4 & 5 & 6 & 7 & 8 & 9 \\
\hline fspring 2: & 1 & 2 & & 9 & 10 & 4 & 5 & 6 & 3 \\
\hline
\end{tabular}

Figure 5. The modified order crossover operator. 
Select three genes at random

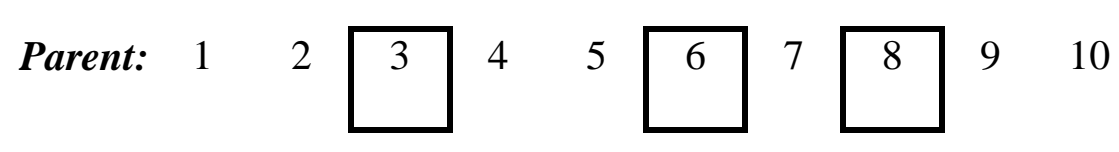

$\begin{array}{lllllllllll}\text { Offspring 1: } & 1 & 2 & \mathbf{3} & 4 & 5 & \mathbf{8} & 7 & \mathbf{6} & 9 & 10 \\ \text { Offspring 2: } & 1 & 2 & \mathbf{6} & 4 & 5 & \mathbf{3} & 7 & \mathbf{8} & 9 & 10 \\ \text { Offspring 3: } & 1 & 2 & \mathbf{6} & 4 & 5 & \mathbf{8} & 7 & \mathbf{3} & 9 & 10 \\ \text { Offspring 4: } & 1 & 2 & \mathbf{8} & 4 & 5 & \mathbf{3} & 7 & \mathbf{6} & 9 & 10 \\ \text { Offspring 5: } & 1 & 2 & \mathbf{8} & 4 & 5 & \mathbf{6} & 7 & \mathbf{3} & 9 & 10\end{array}$

Figure 6. The heuristic mutation operator. 
Selected substring

\begin{tabular}{|c|c|c|c|c|c|c|c|c|c|}
\hline Parent: & 1 & 2 & 3 & 4 & 5 & 6 & 7 & 8 & 9 \\
\hline Offspring: & 1 & 2 & 3 & 7 & 6 & 5 & 4 & 8 & 9 \\
\hline
\end{tabular}

Figure 7. The inversion mutation operator. 


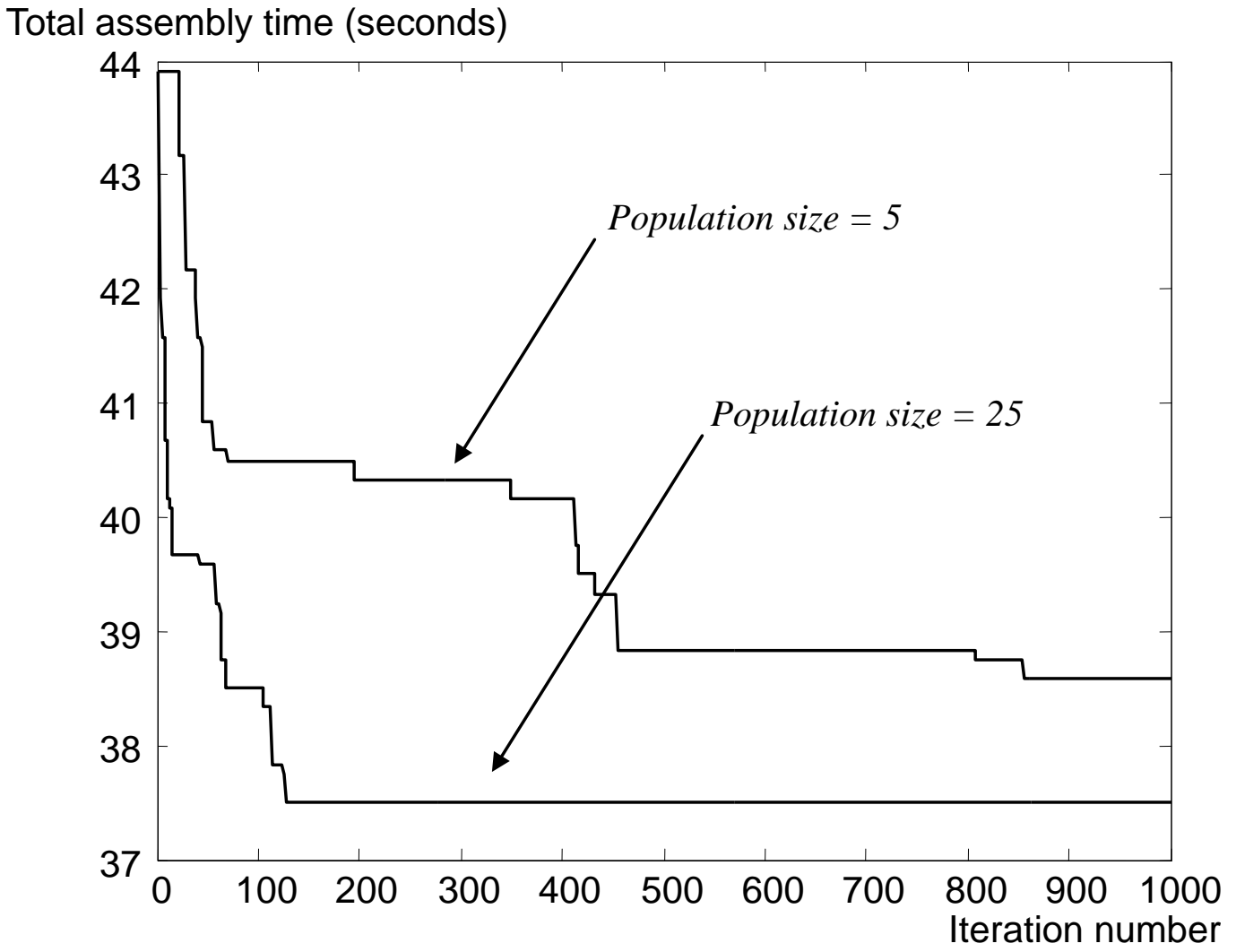

Figure 8 . The effect of population size on the performance of the HGA1. 
Total assembly time (seconds)

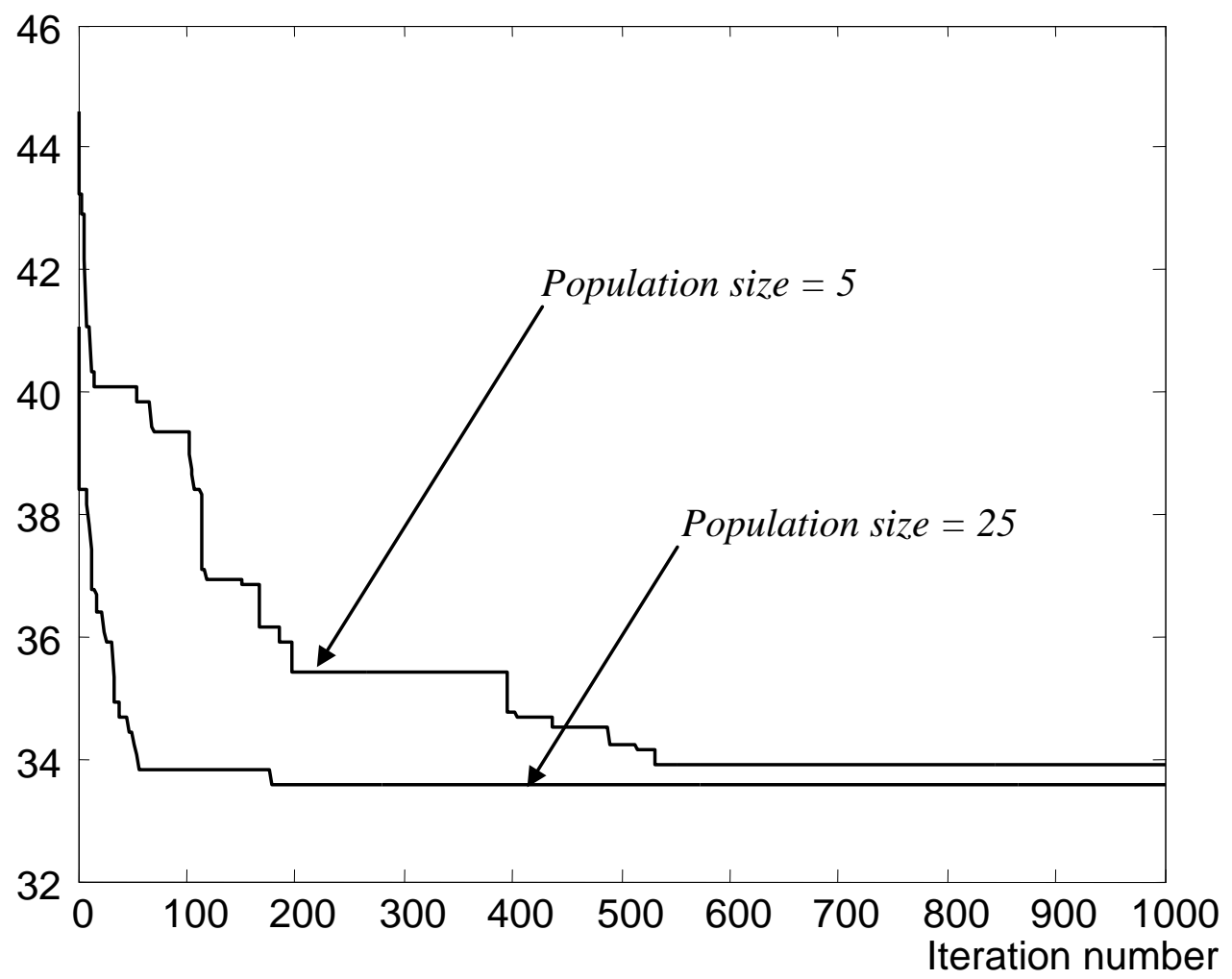

Figure 9. The effect of population size on the performance of the HGA2. 
Table 1. Locations and types of the components.

\begin{tabular}{|c|c|c|c|c|c|c|c|}
\hline \multirow{2}{*}{$\begin{array}{c}\text { Comp. } \\
\text { no. }\end{array}$} & \multicolumn{2}{|c|}{ Locations } & \multirow{2}{*}{$\begin{array}{l}\text { Comp. } \\
\text { types }\end{array}$} & \multirow{2}{*}{$\begin{array}{c}\text { Comp. } \\
\text { no. }\end{array}$} & \multicolumn{2}{|c|}{ Locations } & \multirow{2}{*}{$\begin{array}{c}\text { Comp. } \\
\text { types }\end{array}$} \\
\hline & $x$ & $y$ & & & $x$ & $y$ & \\
\hline 1 & 100 & 60 & 6 & 26 & 180 & 180 & 4 \\
\hline 2 & 100 & 90 & 3 & 27 & 180 & 220 & 8 \\
\hline 3 & 100 & 130 & 2 & 28 & 200 & 60 & 8 \\
\hline 4 & 100 & 180 & 10 & 29 & 200 & 100 & 9 \\
\hline 5 & 100 & 230 & 4 & 30 & 200 & 130 & 9 \\
\hline 6 & 120 & 50 & 4 & 31 & 200 & 140 & 3 \\
\hline 7 & 120 & 90 & 10 & 32 & 200 & 170 & 7 \\
\hline 8 & 120 & 130 & 6 & 33 & 200 & 180 & 10 \\
\hline 9 & 120 & 150 & 9 & 34 & 200 & 220 & 4 \\
\hline 10 & 120 & 190 & 5 & 35 & 220 & 40 & 9 \\
\hline 11 & 120 & 230 & 9 & 36 & 220 & 60 & 9 \\
\hline 12 & 140 & 40 & 9 & 37 & 220 & 100 & 10 \\
\hline 13 & 140 & 80 & 2 & 38 & 220 & 160 & 9 \\
\hline 14 & 140 & 100 & 9 & 39 & 220 & 200 & 7 \\
\hline 15 & 140 & 140 & 4 & 40 & 220 & 220 & 5 \\
\hline 16 & 140 & 180 & 10 & 41 & 240 & 40 & 10 \\
\hline 17 & 140 & 220 & 7 & 42 & 240 & 60 & 9 \\
\hline 18 & 160 & 60 & 5 & 43 & 240 & 80 & 4 \\
\hline 19 & 160 & 100 & 2 & 44 & 240 & 100 & 8 \\
\hline 20 & 160 & 140 & 5 & 45 & 240 & 120 & 1 \\
\hline 21 & 160 & 180 & 4 & 46 & 240 & 140 & 10 \\
\hline 22 & 160 & 220 & 9 & 47 & 240 & 180 & 7 \\
\hline 23 & 180 & 60 & 9 & 48 & 240 & 200 & 6 \\
\hline 24 & 180 & 100 & 5 & 49 & 240 & 210 & 7 \\
\hline 25 & 180 & 140 & 8 & 50 & 240 & 220 & 2 \\
\hline \multicolumn{8}{|c|}{$\begin{array}{l}\text { Remarks: } \\
\text { - } \quad \text { Number of revolver heads }=2 \text {; } \\
\text { - } \quad \text { Indexing time of the head, } t_{r}=0.25 \text { second per index; } \\
\text { - } \quad \text { Average speed of the head, } V_{x}=V_{y}=60 \text { millimeters per second; } \\
\text { - } \quad \text { Coordinates of } H_{A} \text { 's starting point }=(50,100) \text {; } \\
\text { - } \quad \text { Coordinates of } H_{B} \text { 's starting point }=(290,100) \text {. }\end{array}$} \\
\hline
\end{tabular}


Table 2. Locations of the feeders.

\begin{tabular}{|c|c|c|c|c|}
\hline \multirow{2}{*}{} & \multicolumn{2}{|c|}{ Feeder $A$} & \multicolumn{2}{c|}{ Feeder $B$} \\
\cline { 2 - 5 } & $x$ & $y$ & $x$ & $y$ \\
\hline 1 & 0 & 70 & 340 & 70 \\
\hline 2 & 0 & 85 & 340 & 85 \\
\hline 3 & 0 & 100 & 340 & 100 \\
\hline 4 & 0 & 115 & 340 & 115 \\
\hline 5 & 0 & 130 & 340 & 130 \\
\hline 6 & 0 & 145 & 340 & 145 \\
\hline 7 & 0 & 160 & 340 & 160 \\
\hline 8 & 0 & 175 & 340 & 175 \\
\hline 9 & 0 & 190 & 340 & 190 \\
\hline 10 & 0 & 205 & 340 & 205 \\
\hline
\end{tabular}


Table 3. A comparison of the experimental results.

\begin{tabular}{|l|c|c|c|c|}
\hline & \multicolumn{2}{|c|}{ HGA1 (random grouping) } & \multicolumn{2}{c|}{ HGA2 (location grouping) } \\
\hline Population size & 5 & 25 & 5 & 25 \\
\hline $\begin{array}{l}\text { Best one in the initial } \\
\text { population (seconds) }\end{array}$ & 43.9167 & 43.9167 & 44.5833 & 41.0833 \\
\hline $\begin{array}{l}\text { Final best solution } \\
\text { (seconds) }\end{array}$ & 38.5833 & 37.5000 & 33.9167 & 33.5833 \\
\hline $\begin{array}{l}\text { Iteration number for } \\
\text { yielding the final best } \\
\text { solution }\end{array}$ & 855 & 127 & 531 & 178 \\
\hline
\end{tabular}




\section{University Library}

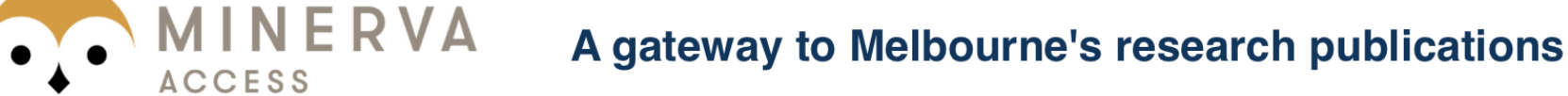

Minerva Access is the Institutional Repository of The University of Melbourne

Author/s:

Ho, W;Ji, P;Wu, Y

Title:

A heuristic approach for component scheduling on a high-speed PCB assembly machine

Date:

2007-12-01

Citation:

Ho, W., Ji, P. \& Wu, Y. (2007). A heuristic approach for component scheduling on a high-speed PCB assembly machine. PRODUCTION PLANNING \& CONTROL, 18 (8), pp.655-665. https://doi.org/10.1080/09537280701602683.

Persistent Link:

http://hdl.handle.net/11343/118686 